\title{
Effects of Alternating Cycle Lengths on Refractoriness of the His-Purkinje System
}

\author{
Stephen Denker, Michael Lehmann, Rehan Mahmud, \\ Carol Gilbert, and Masood Akhtar \\ Natalie and Norman Soref and Family Electrophysiology \\ Laboratory, University of Wisconsin-Mount Sinai Medical \\ Center, Milwaukee, Wisconsin 53201
}

bstract. The effects of alternating cycle lengths (bigeminal rhythm) on His-Purkinje system refractoriness were studied in 14 patients using His bundle and right bundle recordings. Programmed atrial stimulation at constant cycle length (method I) was scanned using the atrial extrastimulus technique (A2) and compared with an atrial cycle length of identical duration coupled to A2 on alternate beats (method II). The results showed that (a) despite shorter cycle length of the His-Purkinje system with method II due to effect of A2 on atrioventricular nodal conduction $(699 \pm 90$ vs. $743 \pm 87 \mathrm{~ms}, P<0.001)$, the relative refractory period of the His-Purkinje system was always longer with method II ( $463 \pm 52$ vs. $440 \pm 43$ $\mathrm{ms}, P<0.001)$. A similar increase also occurred in effective refractory period of the His-Purkinje system; $(b)$ while functional right bundle branch block occurred in eight patients and functional left bundle branch block in two patients with method I, functional right bundle branch block occurred in all 14 patients and left bundle branch block in seven patients with method II; $(c)$ in two patients where both functional right and left bundle branch block occurred with method I this never was manifest at identical degree of abbreviation of His-Purkinje system cycle length but was manifest at identical abbreviation in each of seven patients with method II; $(d)$ site of conduction delay and/or block during functional right bundle branch block was always proximal, i.e., between the His bundle and right bundle recordings with both methods. During method II this resulted in shortening of the subsequent right bundle cycle length relative to the subsequent His bundle (and of necessity left bundle) cycle length.

Received for publication 13 December 1983 and in revised form 29 March 1984.

J. Clin. Invest.

(c) The American Society for Clinical Investigation, Inc. 0021-9738/84/08/0559/12 $\$ 1.00$

Volume 74, August 1984, 559-570
The finding of increased His-Purkinje system refractoriness despite shorter preceding cycle length of the HisPurkinje system during atrial bigeminy has never been previously described and suggests that classical concepts of His-Purkinje system behavior may require revision in this setting. Secondly, during atrial bigeminy the occurrence of alternating functional bundle branch block cannot be accounted for solely by the degree of abbreviation of His-Purkinje system cycle length, but may be explained by a relative shortening of the next ipsilateral bundle branch cycle length in the bundle manifesting block.

\section{Introduction}

Functional conduction delay or block within the His-Purkinje system (HPS) ${ }^{1}$ during propagation of premature atrial (A2) impulses is a well recognized phenomenon that may manifest as right (R) or left bundle branch block (LBBB) or bilateral block within the HPS (1-13). These patterns of aberrant ventricular conduction (VAb) or block within the HPS are contingent upon HPS refractory period properties, which are known to be cycle length $(\mathrm{CL})$ dependent, varying directly with $\mathrm{CL}$ preceding $\mathrm{A} 2$ $(4,9,13)$. Previous studies have also shown that different degrees of $\mathrm{CL}$ abbreviation are necessary for manifestation of RBBB vs. LBBB and this has been attributed to differences in refractoriness between the RBB and LBB (3).

Considering these concepts, there exists in the literature an unexplained paradox concerning VAb. In a report by Cohen et al. (4) on VAb in man, the authors suggested that an atrial bigeminal rhythm, whereby $A 2$ was coupled to every sinus beat, increased the likelihood of VAb by producing a prolonged $\mathrm{CL}$ following A2. Interestingly, it was also observed that such a pacing method produced alternating patterns of VAb with each

1. Abbreviations used in this paper: $\mathrm{AV}$, atrioventricular; $\mathrm{BB}$, bundle branch; BBB, BB block; BCL, basic CL; CL, cycle length; ECG, electrocardiogram; ERP, effective refractory period; FRP, functional refractory period; HB, His bundle; HV, His-ventricular; HPS, His-Purkinje system; LBBB, left BBB; RB, right bundle; RB-V, RB-ventricular, RBBB, right $\mathrm{BBB}$; RRP, relative refractory period; $\mathrm{VAb}$, aberrant ventricular conduction. 
A2 (i.e., RBBB alternating with LBBB) despite identical A2 coupling intervals. This would be a distinctly unusual finding, if indeed, the HPS CL changes were also identical. While the role of changes in the cycle lengths of the bundle branches (BBs) during alternate patterns of VAb have been postulated $(5,11)$ this has never previously been demonstrated in man.

In an attempt to explain this phenomenon of alternating patterns of functional BBB, the present study used both His bundle (HB) and right bundle (RB) recordings and a programmed atrial stimulation specifically designed protocol to permit identical CL sequences. Moreover, in view of recent findings concerning the effect of abrupt alterations in atrial CL on refractoriness of the HPS (14), this study attempted to determine if the alternating CL changes per se, rather than prolonged CL (as occurs with A2 coupled to sinus beats), contributes to the increased likelihood of VAb.

\section{Methods}

Electrophysiologic studies were performed in the unsedated, postabsorptive state after obtaining signed consent to the explained procedure (15). Using local anesthesia and fluoroscopic guidance, multipolar electrode catheters were percutaneously introduced and positioned in the high right atrium, the atrioventricular (AV) junction, and the right ventricle. The simultaneous recordings from the $\mathrm{HB}$ and $\mathrm{RB}$ were obtained either with a single quadripolar catheter (interelectrode distance $1 \mathrm{~cm}$ ) or with two separate electrode catheters. All intracardiac electrograms (filtered at 30-500 Hz), three surface electrocardiographic leads (I, II, and VI), and time lines were simultaneously displayed on a multi-channel oscilloscope and recorded on magnetic tape for later reproduction. Electrical stimulation was performed with a digital stimulator capable of delivering rectangular impulses of variable voltage and duration with total current $<3 \mathrm{~mA}$. In none of the patients was the magnitude or duration of the electrical impulse altered during the study. All equipment was grounded and an isolation unit was used for electrical stimulation.

Patients were studied with the conventional technique (method I) of atrial premature stimulation (A2) wherein the $\mathrm{A} 2$ was introduced after a series of six atrial beats of predetermined constant cycle length (A1A1). Scanning of constant atrial cycle length with A2 was initiated at relatively long $A 1 A 2$ intervals which were outside the relative refractory period (RRP) of the HPS and were decreased by $10 \mathrm{~ms}$ intervals until functional refractory period (FRP) of the AV node or atrial refractoriness was encountered.

With method II premature atrial stimulation was coupled to an atrial $\mathrm{CL}$ of identical duration as used in method $\mathrm{I}$, however, every other beat was premature producing a bigeminal rhythm. This method used the same initial six-beat atrial drive of constant $C L$ and began the alternating CL pattern with A2 of method I. As shown in Fig. 1 this results in the designation of the $C L$ in method II as $A 2 A 1$, which equals $A 1 A 1$ in method $\mathrm{I}$. This $\mathrm{CL}$ was scanned with $\mathrm{A} 2$ in a similar fashion as during method I.

Definition of terms $(14,16,17)$. The His-ventricular (HV) and RBventricular (RB-V) intervals were measured from the onset of respective deflections to the earliest detectable ventricular activity, whether recorded on surface electrocardiogram (ECG) or one of the local electrograms. RRP of the HPS is the longest $\mathrm{H} 1 \mathrm{H} 2$ interval at which $\mathrm{H} 2$ conducts to the ventricles with a longer HV interval than the basic drive beat or with a QRS showing a definite BBB pattern. Effective refractory period
(ERP) of the HPS is the longest $\mathrm{H} 1 \mathrm{H} 2$ interval at which $\mathrm{H} 2$ does not propagate to the ventricles.

Patient population. All patients included in this study were in sinus rhythm with normal intraventricular conduction on resting ECG, had normal atrium-His and HV intervals, and manifested VAb with atrial premature stimulation during one or both methods of stimulation described above. The 14 consecutive patients (nine males, five females) were 23-68 years old (mean 45 \pm 13 ) and were studied for various reasons, predominantly for palpitations, syncope, or ventricular tachychardia. The underlying structural heart disease was arteriosclerotic in six, mitral valve prolapse in one, valvular in one, while the remaining had no clinically evident heart disease. No patient in this series was taking cardioactive medications within 48-72 h before the study. In nine patients, satisfactory RB recordings were obtained $(16,17)$ and used to determine the site of functional delay and/or block along the H-RB axis (17).

Statistical analysis. Mean values are expressed with corresponding standard deviations. Comparison of mean values was done using the paired $t$ test. Statistical significance was defined at the 5\% level.

\section{Results}

The electrophysiologic data are listed in Tables I-III. For the 14 patients in this study the atrial basic cycle length (BCL) used was $743 \pm 87 \mathrm{~ms}$ (range $650-900 \mathrm{~ms}$ ). The HV interval ranged from 40 to $55 \mathrm{msec}$ (mean $48 \pm 5 \mathrm{~ms}$ ). In the nine patients (Nos. 1-9) where $R B$ recordings were obtained, the $H-R B$ ranged from 20 to $35 \mathrm{~ms}$ (mean 27 \pm 6 ) and the RB-V ranged from 10 to $30 \mathrm{~ms}$ (mean $21 \pm 7$ ).

Effect of alternating changes in atrial CLs on $H-H C L$. Since the atrial drive was constant during method I, A1Al equaled H1H1. However, during method II, the atrial BCL (designated A2A1) was always longer than $\mathrm{H} 2 \mathrm{H} 1$ due to the effect on $\mathrm{AV}$ nodal conduction of A2 (Table I, Fig. 1) (14). Therefore, the $\mathrm{H}-\mathrm{H}$ interval coupled to $\mathrm{A} 2$ was longer in method $\mathrm{I}$ than in method II ( $743 \pm 87$ vs. $699 \pm 90 \mathrm{~ms}, P<0.001)$. For a given atrial $\mathrm{BCL}$ and $\mathrm{A} 2$ during method II, the subsequent $\mathrm{H} 2 \mathrm{H} 1$ intervals and $\mathrm{H} 1 \mathrm{H} 2$ intervals remained constant. These effects are shown in Fig. $1 A$ where the atrial bigeminal rhythm is preceded by a constant atrial drive, (the last three beats of which are the initial complexes in this panel). Note that at constant atrial $\mathrm{CL}$ A1A1 $=\mathrm{H} 1 \mathrm{H} 1$, but during atrial bigeminy $\mathrm{A} 2 \mathrm{~A} 1$ $>\mathrm{H} 2 \mathrm{H} 1$ due to $\mathrm{A} 2 \mathrm{H} 2$ delay.

Effect of alternating changes in CL on HPS refractoriness (Table I). An increase in HPS refractoriness occurred in all patients with method II compared with method I despite the shorter $\mathrm{H}-\mathrm{H}$ CL coupled to A2 with method II described above. This is illustrated in Figs. 2 and 3, which show the onset of functional RBBB, LBBB, and bilateral block within the HPS with method I compared with method II. The data for the same patient is plotted for both methods in Fig. 4, which graphically depicts the longer RRP and ERP HPS with method II. While RRP HPS was encountered in all patients with method II, in six patients RRP HPS was less than FRP AV node with method I, and therefore, was not encountered with this method. During method I the FRP AV node in these patients, however, was less 

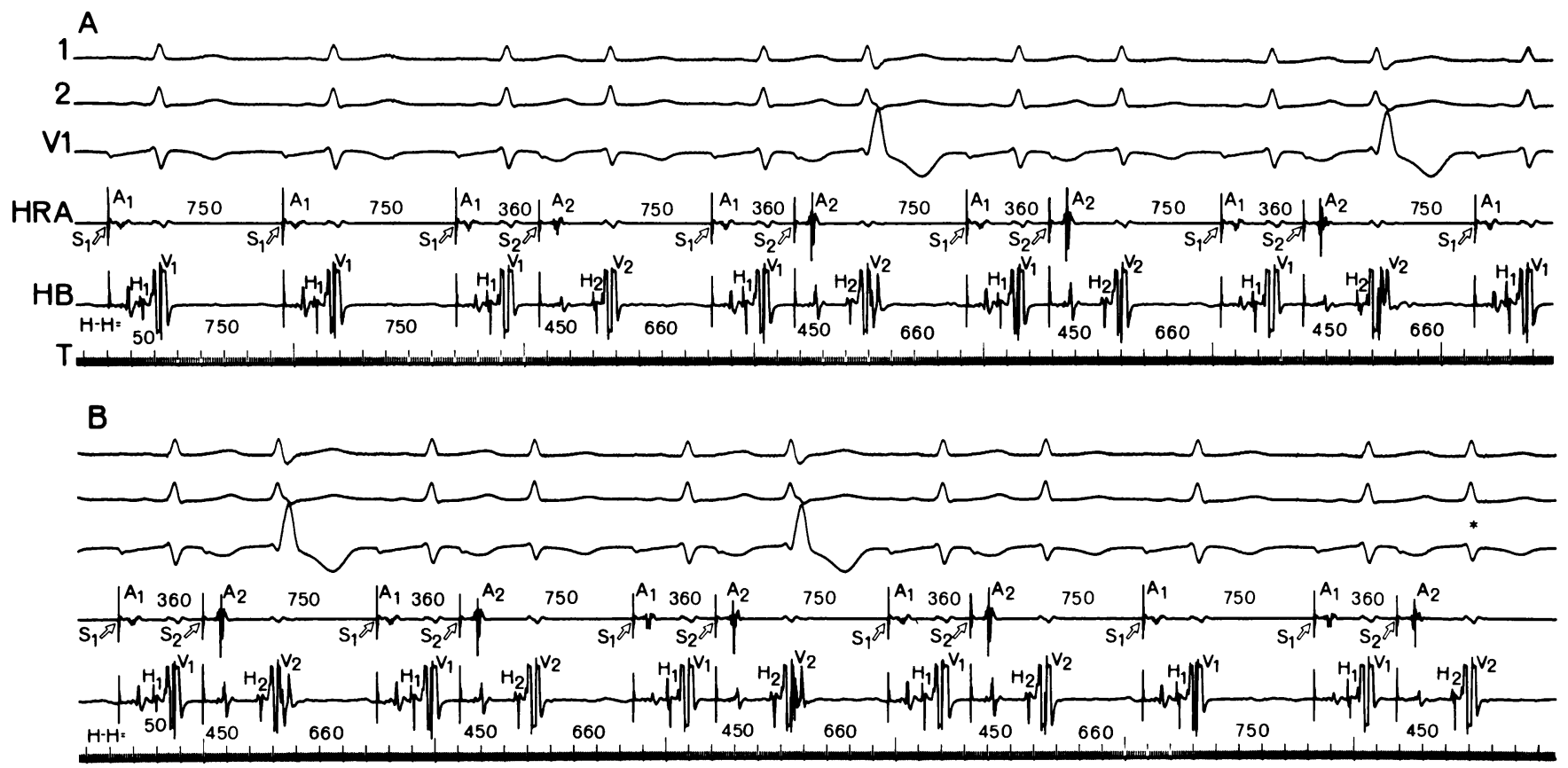

Figure 1. The tracings from patient No. 10 illustrate the pacing methods used $(A)$, the effect of alternating changes in atrial CL on the subsequent "long" H-H interval, the increase in RRP HPS with this method despite shorter "long" $\mathrm{H}-\mathrm{H}$ interval preceding $\mathrm{A} 2$, and the alternation of RBBB pattern VAb with no VAb at identical $\mathrm{H} 1 \mathrm{H} 2$ intervals. Note the last three beats of the constant atrial CL drive (first three beats of $A$ ) have a constant H-H CL equal to atrial drive of $750 \mathrm{~ms}$ (method I). An A1A2 of $360 \mathrm{~ms}$ produces an H1H2 of $450 \mathrm{~ms}$ and impulse propagation with essentially normal intraventricular conduction. Subsequent alternating long-short atrial CLs with identical intervals (method II) result in a long H-H interval of only $660 \mathrm{~ms}$, a decrease of $90 \mathrm{~ms}$ compared with constant CL. This occurs due to increase in AV nodal conduction time with A2. Nonetheless, at identical $\mathrm{H} 1 \mathrm{H} 2$, VAb with RBBB occurs with the next A2.

than the RRP HPS during method II. For all 14 patients the RRP HPS increased from $440 \pm 43$ ms with method I to $463 \pm 52$ ms $(P<0.001)$ with method II (using FRP AV node values where RRP HPS was not encountered). The ERP HPS was manifest in four patients with both methods. For each of these cases ERP HPS was longer with method II compared with method $I$ and the mean values increased from $435 \pm 23$ to $454 \pm 20$ $\mathrm{ms}(P<0.025)$ (compare Fig. $5 D$ to Fig. $6 B)$.

Effect of alternating changes in CL on patterns of $V A b$ (Tables $I$ and II). All patients who manifested RBBB and/or LBBB VAb with method I also manifested the same pattern VAb with method II. However, while eight patients had RBBB and two had LBBB with method I, 14 had RBBB and seven had LBBB with method II. Remarkably, while two patients had both RBBB and LBBB with method $I$, this occurred in seven with method II. Moreover, while it was never observed that RBBB and LBBB occurred at the same $\mathrm{H} 1 \mathrm{H} 2$ with method I, RBBB and LBBB
(Note that the long CLs during alternating CLs are labeled A2A1, $\mathrm{H} 2 \mathrm{H} 1, \mathrm{~V} 2 \mathrm{~V} 1$.) However, with the next long-short $\mathrm{CL}$ sequence VAb is abolished despite identical $\mathrm{H} 2 \mathrm{H} 1$ and $\mathrm{H} 1 \mathrm{H} 2$ where previously functional RBBB occurred. Alternation of no VAb and functional RBBB with alternate $A 2$ persists until in $B$ (continuous with $A$ ) the alternating CL of method II is changed and two atrial CLs of $750 \mathrm{~ms}$ are programmed and coupled to the identical A2 of $360 \mathrm{~ms}$ (last three CLs in $B$ ). Note again that despite the again longer $\mathrm{H}-\mathrm{H}$ interval of $750 \mathrm{~ms}$ no VAb occurs. The beat indicated by asterisk depicts the next expected occurrence of functional RBBB with A2 during alternating long-short CLs, which has been abolished by two beats of constant CL. 1, 2, V1, Surface ECG leads; HRA, High right atrial electrogram; HB, His bundle electrogram; T, time lines. All measurements are in milliseconds.

occurred at identical $\mathrm{H} 1 \mathrm{H} 2$ in all seven patients with method II. Examples of this phenomenon are shown in Fig. $3 C$ and Fig. 6, $A$ and $C$.

Relation between patterns of $V A b$ and HPS conduction (Tables I-III). VAb and HPS conduction could be evaluated in terms of $\mathrm{HV}$ conduction in all patients and $\mathrm{H}-\mathrm{RB}$ and $\mathrm{RB}-\mathrm{V}$ conduction in nine patients (Nos. 1-9) where RB recordings were available. With method $I, 4 / 8$ patients during $R B B B$ and 2/2 during LBBB manifested HV prolongation compared with sinus beats (Table I). Different magnitudes of $\mathrm{HV}$ prolongation during $\mathrm{RBBB}$ and $\mathrm{LBBB}$ occurred in $2 / 2$ patients where both patterns of VAb occurred (Fig. 2, $C$ and $D$ ). With method II, $3 / 14$ patients during $\mathrm{RBBB}$ and $7 / 7$ patients during $\mathrm{LBBB}$ manifested HV prolongation (Table I). During this latter method the occurrence of alternating RBBB and $\mathrm{LBBB}$ at identical $\mathrm{H} 1 \mathrm{H} 2$ (7 patients) was associated with $\mathrm{HV}$ prolongation with both patterns of VAb (in 3 patients) or during LBBB solely in the 
Table I. HPS Refractoriness and Conduction during Methods I and II

\begin{tabular}{|c|c|c|c|c|c|c|c|c|c|c|}
\hline \multirow[b]{3}{*}{ Patient } & \multicolumn{6}{|l|}{ Method I } & \multicolumn{4}{|l|}{ Method II } \\
\hline & \multirow{2}{*}{$\frac{B C L}{A|A|=H|H|}$} & \multirow[b]{2}{*}{ HV } & \multicolumn{3}{|c|}{ Type of block HPS (longest H1H2) } & \multirow{2}{*}{$\begin{array}{l}\text { FRP } \\
\text { AVN }\end{array}$} & \multirow{2}{*}{$\frac{\mathrm{BCL}}{\mathrm{A} 2 \mathrm{~A} 1 / \mathrm{H} 2 \mathrm{HI}}$} & \multicolumn{3}{|c|}{ Type of block HPS (Longest HIH2) } \\
\hline & & & RBBB (HV) & LBBB (HV) & Bilateral & & & RBBB (HV) & LBBB (HV) & Bilateral \\
\hline \multicolumn{11}{|l|}{ No. } \\
\hline \multirow[t]{2}{*}{1} & 700 & 50 & $480(70)$ & - & 455 & 440 & $700 / 680$ & $520(50)$ & $520(70)$ & \\
\hline & & & & & & & 660 & & & 470 \\
\hline 2 & 800 & 45 & - & - & - & 490 & $800 / 750$ & $510(45)$ & - & - \\
\hline 3 & 700 & 55 & $420(70)$ & - & - & 410 & $700 / 640$ & $430(55)$ & $430(70)$ & - \\
\hline 4 & 650 & 55 & - & - & - & 380 & $650 / 620$ & $385(70)$ & $385(80)$ & - \\
\hline 5 & 700 & 50 & - & - & - & 370 & $700 / 610$ & $400(50)$ & - & - \\
\hline 6 & 600 & 45 & $370(45)$ & - & - & 340 & $600 / 580$ & $405(45)$ & - & - \\
\hline 7 & 800 & 45 & - & - & - & 420 & $800 / 760$ & $440(45)$ & - & - \\
\hline \multirow[t]{3}{*}{8} & 700 & 45 & $450(60)$ & - & 420 & 390 & $700 / 670$ & $465(45)$ & $465(60)$ & \\
\hline & & & & & & & 670 & & & \\
\hline & & & & & & & 670 & $425(90)$ & $425(60)$ & 455 \\
\hline \multirow[t]{3}{*}{9} & 700 & 45 & $450(45)$ & $420(125)$ & 410 & 410 & $700 / 680$ & $480(45)$ & - & \\
\hline & & & & & & & 670 & $450(45)$ & $450(105)$ & \\
\hline & & & & & & & 670 & & & 420 \\
\hline 10 & 750 & 50 & - & - & - & 420 & $750 / 660$ & $450(50)$ & - & - \\
\hline 11 & 700 & 40 & $390(80)$ & $380(100)$ & - & 380 & $700 / 640$ & $430(40)$ & $430(70)$ & - \\
\hline \multirow[t]{2}{*}{12} & 800 & 50 & - & - & - & 480 & $800 / 780$ & $570(50)$ & - & - \\
\hline & & & & & & & 770 & $520(60)$ & $520(80)$ & \\
\hline \multirow[t]{2}{*}{13} & 900 & 40 & $480(40)$ & - & 455 & 445 & $900 / 850$ & $495(40)$ & - & \\
\hline & & & & & & & 850 & & & 465 \\
\hline 14 & 900 & 50 & $460(50)$ & - & - & 425 & $900 / 875$ & $495(50)$ & - & - \\
\hline
\end{tabular}

AVN, AV node. All electrophysiologic data are given in milliseconds.

remaining patients (Tables I and II). In the three cases with $\mathrm{HV}$ prolongation during both patterns, $\mathrm{HV}$ prolongation with $\mathrm{LBBB}$ was 10-20 ms longer compared with RBBB. However, in one patient where conduction resumed at shorter $\mathrm{H} 1 \mathrm{H} 2$ after $\mathrm{BBH}$ (gap within HPS) (10) HV prolongation with RBBB exceeded that with LBBB (Fig. 6, $A$ and $C$ ). In nine cases where RB recordings were available (Table III) the $R B$ potential disappeared in all patients manifesting $\mathrm{RBBB}$, indicating conduction delay and/or block proximal to or within the area of $\mathrm{RB}$ recording (17) with both methods I and II. An example of this is shown in Fig. $2 B$ for method $I$ and Fig. $3 A$ for method II. During LBBB with $\mathrm{HV}$ prolongation the RB-V intervals were comparable to sinus beats, again suggesting presence of conduction delay proximal to $\mathrm{RB}$ recording site. This is demonstrated in Fig. $2 D$ and Fig. $3 C$.
Relationship between H1HI (H2H1) CL and RB1RBI (and RB2RBI) $C L$ and $V A b$ (Table III). During method I using constant BCL, H1H1 equaled to RB1RB1. However, during method II H2H1 equaled RB2RB1 only where the "long" CL followed no VAb. Because of the proximal site of conduction delay during VAb as mentioned above, the "long" CL following VAb had a shorter RB-RB CL compared with H-H CL, although the exact shortening in RB-RB CL could not be determined following RBBB. The following relationships were therefore seen: (a) where RBBB alternated with no VAb (Fig. $3 A$ ), the $\mathrm{H} 2 \mathrm{H} 1$ CLs (long H-H CLs) were identical preceding premature beats conducted normally and with RBBB pattern, however RB2RB1 was longer preceding RBBB compared with no VAb (Table III, Fig. $3 A$ ); (b) where RBBB alternated with LBBB, the H2H1 CLs preceding $\mathrm{R}$ and LBBB were identical (Fig. $3 C$, Fig. 6, $A$ 


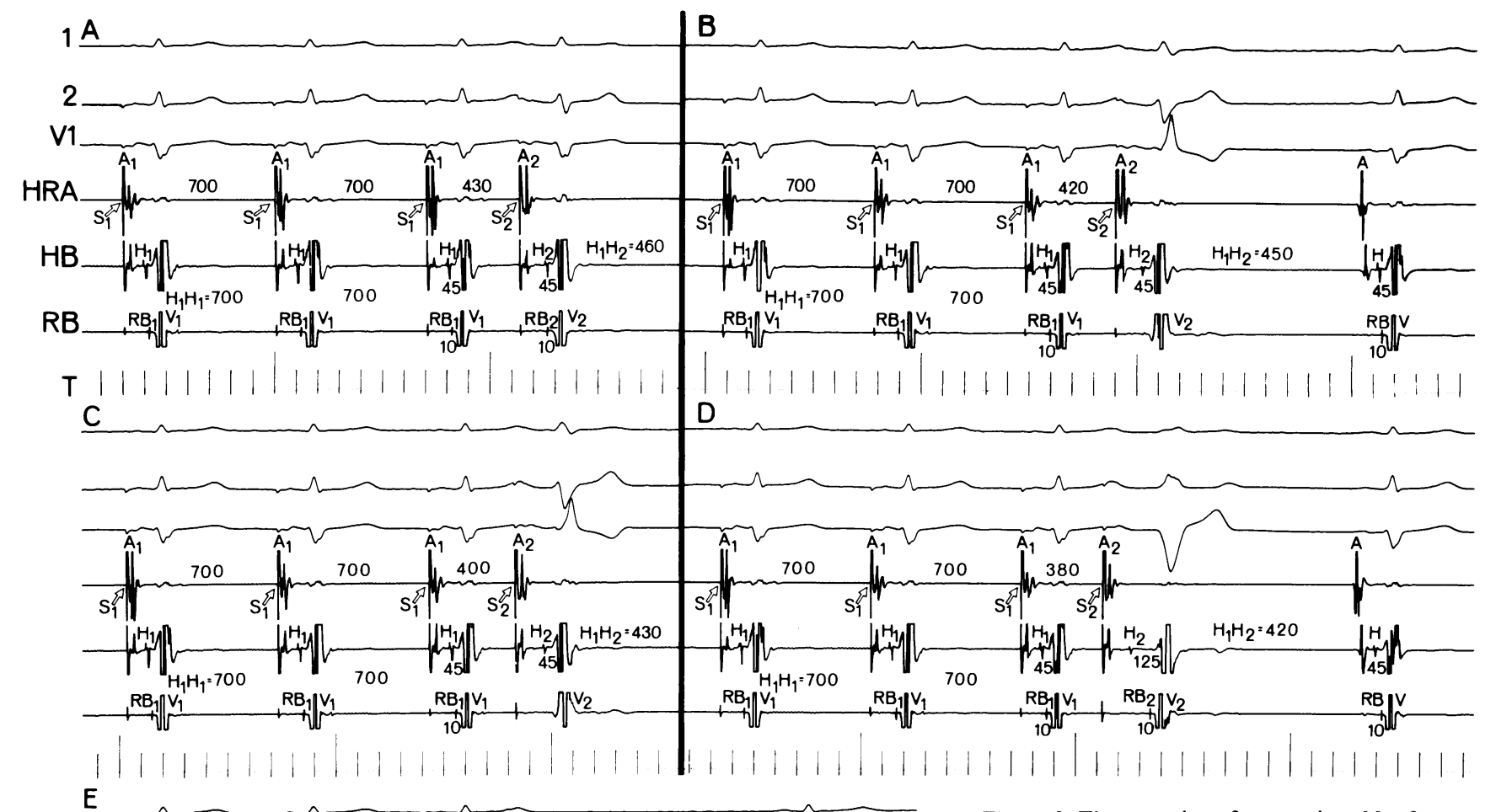
E

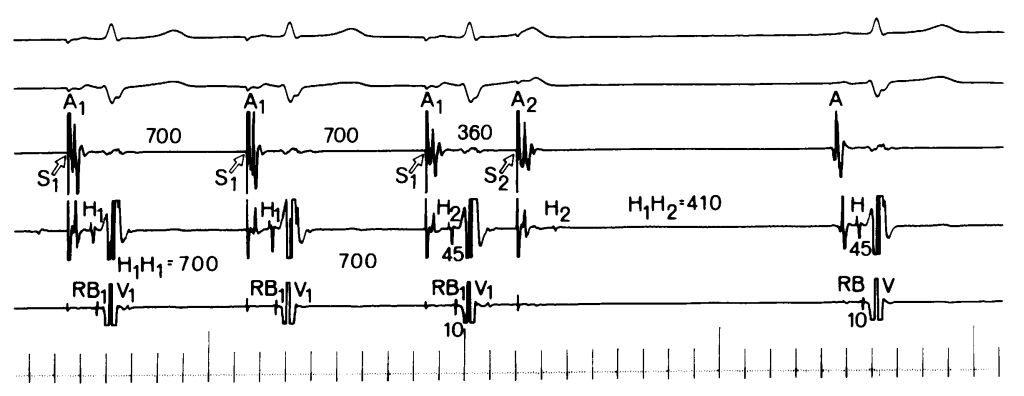

Figure 2. These tracings from patient No. 9 show the effect of progressive decrease in A1 A2 on VAb and HPS conduction during method $\mathrm{I}$. For all panels constant BCL is $700 \mathrm{~ms}$. In $A$, A1A2 of $430 \mathrm{~ms}$ results in $\mathrm{H} 1 \mathrm{H} 2$ of $460 \mathrm{~ms}$ and no VAb. H2V2 and RB2V2 are 45 and $10 \mathrm{ms,}$ respectively, and are identical to sinus reference beats. With a decrease in $\mathrm{H} 1 \mathrm{H} 2$ interval to 450 $\mathrm{ms}$, functional RBBB occurs in $B$. Note that while the $\mathrm{H} 2 \mathrm{~V} 2$ has remained at $45 \mathrm{~ms}$, no RB2 deflection is seen, suggesting conduction delay and/or block in the H-RB axis before or at RB recording site. Further abbreviation of $\mathrm{H} 1 \mathrm{H} 2$ interval shows onset of functional LBBB at $420 \mathrm{~ms}(D)$. Note that this is associated with $\mathrm{H} 2 \mathrm{~V} 2$ of $125 \mathrm{~ms}$, but RB2V2 is equal to sinus reference beats. This suggests bilateral delay, or delay and block along the H-RB and H-LB axis, respectively. Again, delay in H-RB axis appears to be proximal. $E$ shows the occurrence of bilateral block below the HB but proximal to the RB (i.e., no RB2 deflection) with further H1H2 shortening. 1, 2, V1, surface ECG leads; HRA, high right atrial electrogram; HB, His bundle electrogram; RB, right bundle electrogram; T, time lines.

and $C$ ), however RB2RB1 CL preceding RBBB was greater than RB2RB1 CL preceding LBBB where greater magnitude HV prolongation occurred with RBBB (Fig. $6 C$ ). When greater magnitude of HV prolongation occurred with LBBB (as was usually the case) $R B 2 R B 1 C L$ preceding $R B B B$ was greater than RB2RB1 CL preceding LBBB as long as the difference between magnitudes of $\mathrm{HV}$ prolongation associated with $\mathrm{RBBB}$ and LBBB was less than RB-V interval during LBBB (Fig. $6 \mathrm{~A}$ ); where this difference was greater than RB-V interval it could not be definitely stated that RB2RB1 CL preceding RBBB was greater than RB2RB1 CL preceding LBBB (Fig. $3 \mathrm{C}$ ). Nonetheless, if a similar pattern of conduction delay and/or block observed in the H-RB axis occurs in the H-LB axis, as previous studies suggest $(3,18,19)$ then the least one can assume during alternating $R B B B$ and $L B B B$ is that $R B 2 R B 1$ is greater than LB2LB1 preceding RBBB, and LB2LB1 is greater than RB2RB1 preceding LBBB.

\section{Discussion}

Alternating functional RBBB and LBBB is a commonly observed phenomenon during atrial premature stimulation coupled to every sinus beat. Whereas it has been postulated that this phe- 

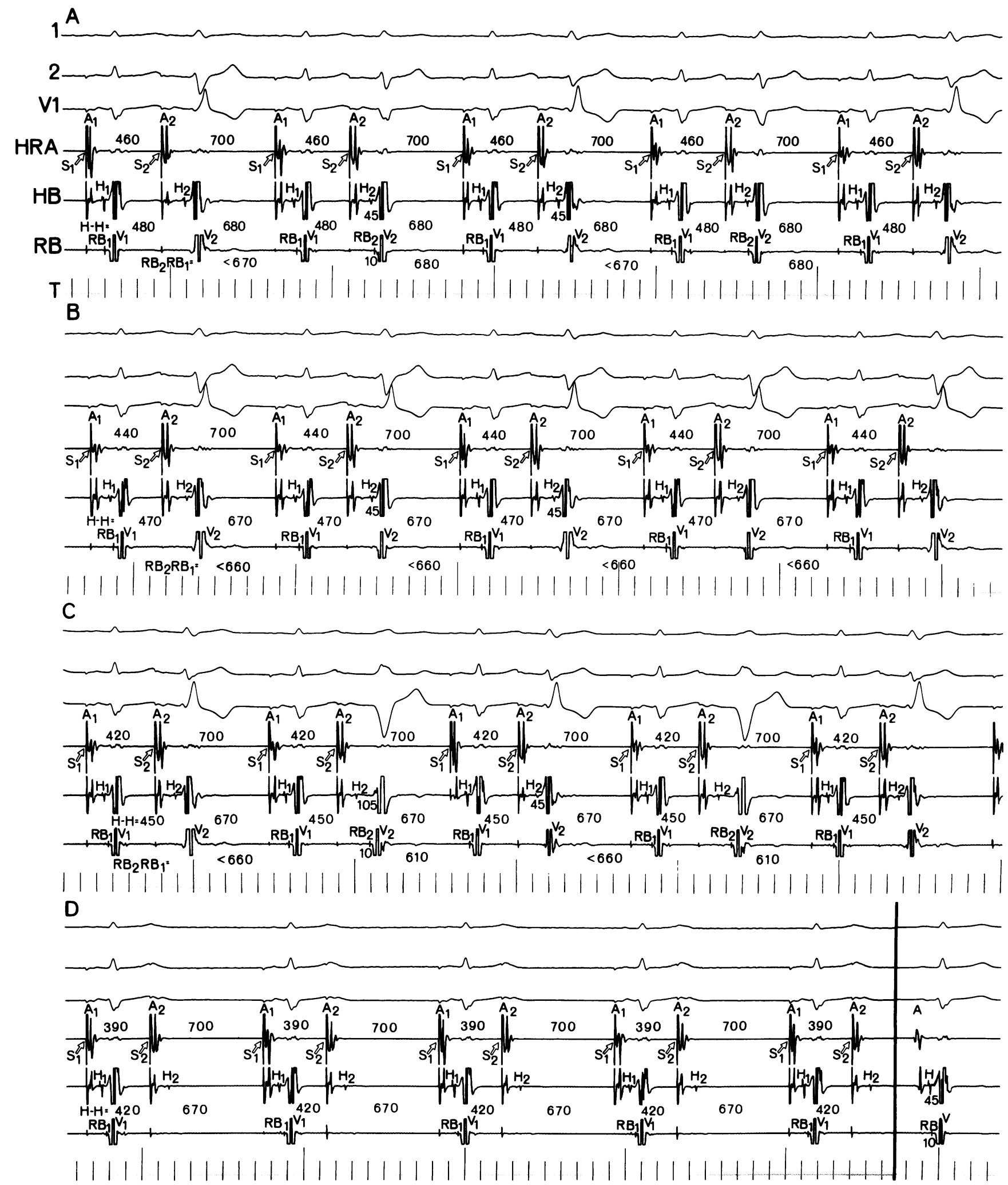


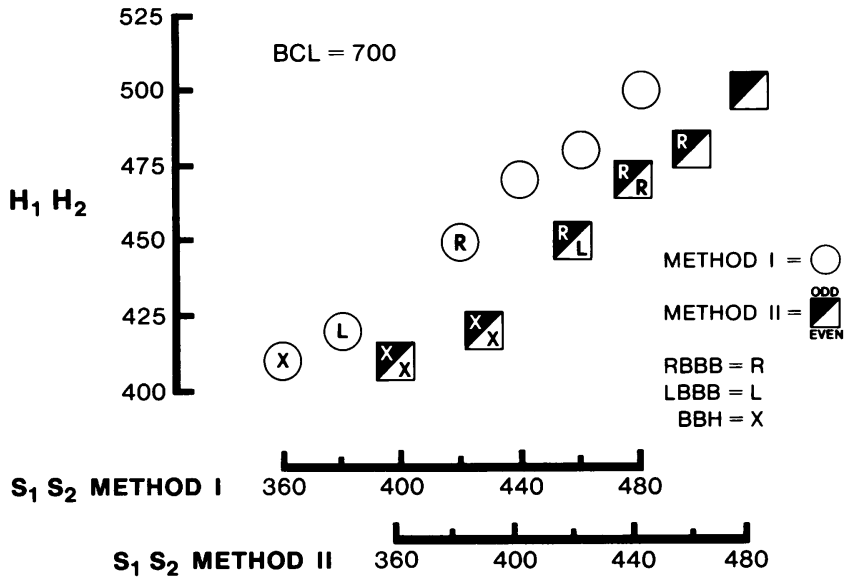

Figure 4. A plot of the data from patient No. 9 (same patient as in Figs. 2 and 3). For the most part the legends are self explanatory. However, it should be noted that the absence of letters in circles or squares indicate normal intraventricular conduction. For method II since different patterns of $\mathrm{VAb}$ can occur at identical $\mathrm{H} 1 \mathrm{H} 2$, the black half of each square represents all odd numbered A2 (i.e., 1st, $3 r d, 5$ th) while the white half represents all even numbered $A 2$ (i.e., 2nd, 4th, 6th). Note that compared with method I, method II results in onset of functional RBBB, functional LBBB, and block below His $(\mathrm{BBH})$ at longer $\mathrm{H} 1 \mathrm{H} 2$. Also during method I, normal intraventricular conduction, functional RBBB, and functional LBBB occur at different $\mathrm{H} 1 \mathrm{H} 2$ intervals, however, this is not the case with method II.

nomenon may be related to changes in BB CL (5), the underlying electrophysiologic mechanism has never been systematically investigated or explained. Earlier studies (4) on VAb in man have suggested that such a bigeminal rhythm can increase the likelihood of VAb. This was attributed to the longer attainable CL such a method produces with sinus escape beats following each A2. However, the effect of alternating CLs on HPS refractoriness has never been previously examined.

The present study attempted to specifically address these issues using programmed atrial stimulation whereby the identical "long" atrial CL coupled to A2 was scanned with A2 during a conventional method of constant CL (method I) and during atrial bigeminal rhythm (method II). The findings demonstrate: (a) an increase in HPS refractoriness with method II compared with method I despite the fact that the $\mathrm{H}-\mathrm{H}$ cycle length preceding A2 is shorter with method II compared with method I; and (b) during atrial bigeminal rhythm alternating patterns of VAb (i.e., RBBB alternating with LBBB) can occur at identical degrees of HPS CL abbreviation, as can VAb alternating with normal intraventricular conduction (i.e., RBBB alternating with no VAb). These results could not have been predicted by classical electrophysiologic concepts of HPS behavior whereby HPS refractoriness is CL-dependent varying directly with $\operatorname{CL}(4,9,13,20)$.

The present finding of increased HPS refractoriness with atrial bigeminal rhythm compared with constant $C L$ is consistent, however, with our previously reported observation of increased HPS refractoriness with abrupt short-to-long CL changes (14, $21,22)$. Results from these studies suggested that the HPS responds in a dynamic fashion to the rate and direction of $\mathrm{CL}$ change and not solely to the absolute duration of preceding CL or cumulative duration of preceding CLs. This dynamic nature of the HPS response is again seen in the present study where alternating long-short $\mathrm{CL}$ changes are used compared with the previous study using a basic drive of constant CL abruptly increased or decreased for one beat before A2. Remarkably, the appearance of VAb during alternation of atrial $\mathrm{CL}$ and disappearance after two constant atrial CL (Fig. 1) attests to the rapidity with which these dynamic changes occur. Conceivably, alternating $C L$ changes produce greater rates of $C L$ change and may have greater effect on HPS refractoriness than a single comparable CL change (22). While this hypothesis cannot be conclusively proven it is nonetheless evident that such $\mathrm{CL}$ changes have a pronounced effect on HPS behavior that needs to be considered when CLs are not constant.

The second finding of alternating patterns of VAb at identical degrees of HPS CL abbreviation is perhaps even more fascinating and has never before been described. Any proposed explanation for this phenomenon will have to explain the various patterns
Figure 3. These tracings show the effect of alternating atrial CLs (method II) on HPS refractoriness and conduction in the same patient as in Fig. 2. The same "long" atrial CL of $700 \mathrm{~ms}$ is used as in Fig. 2. Note, however, that due to effect of A2 on AV nodal conduction the $\mathrm{H} 2 \mathrm{H} 1$ (long $\mathrm{H}-\mathrm{H} \mathrm{CL}$ ) is decreased (to $680 \mathrm{~ms}$ in $A$ and to $670 \mathrm{~ms}$ in $B-D$ ). Despite the shorter $\mathrm{H} 2 \mathrm{H} 1$ interval the onset of functional RBBB occurs at an $\mathrm{H} 1 \mathrm{H} 2$ of $480 \mathrm{~ms}(A)$ compared with $450 \mathrm{~ms}$ during constant $\mathrm{CL}$ (Fig. 2). While the $\mathrm{H} 2 \mathrm{~V} 2$ during functional RBBB remains at equal to reference sinus beats, the RB2 deflection is not present during functional RBBB similar to what $O C-$ curred during method I. Note the occurrence of alternation of functional RBBB with no VAb with every other A2 despite identical $\mathrm{H} 2 \mathrm{H} 1$ and $\mathrm{H} 1 \mathrm{H} 2$. However, due to delayed RB depolarization during functional RBBB, the RB2RB1 CL preceding $A 2$ with no VAb is expected to be shorter than the RB2RB1 CL preceding $A 2$ with functional RBBB. (The "<" value is measured from onset of V2 to next
RB1). In $B$, further abbreviation of $\mathrm{H} 1 \mathrm{H} 2$ results in functional RBBB with each $A 2$. Again the H2V2 is equal to reference sinus beats, suggesting no conduction delay along the contralateral $\mathrm{BB}$, and the $\mathrm{RB} 2$ deflection is not present. Abbreviation of $\mathrm{H} 1 \mathrm{H} 2$ to $450 \mathrm{~ms}(C)$ produces alternating functional RBBB and LBBB. Note the occurrence of functional LBBB at an $\mathrm{H} 1 \mathrm{H} 230 \mathrm{~ms}$ longer compared with constant CL (Fig. $2 D$ ). Similar to the occurrence in $A$ of functional RBBB alternating with no VAb with identical $\mathrm{H} 2 \mathrm{H} 1$ and $\mathrm{H} 1 \mathrm{H} 2$ intervals, $C$ shows functional RBBB and LBBB at identical $\mathrm{H} 2 \mathrm{H} 1$ and $\mathrm{H} 1 \mathrm{H} 2$ intervals. During functional LBBB there is HV prolongation with no change in RB2V2 interval compared with reference beat (in $D$ ) and that with functional RBBB there is no change in $\mathrm{H} 2 \mathrm{~V} 2$ interval but RB2 deflection is not present compared with reference beat. In $D$ bilateral block below HB occurs at an $\mathrm{H} 1 \mathrm{H} 2$ of $420 \mathrm{~ms}, 10 \mathrm{~ms}$ longer than during method I (Fig. $2 E$ ). 

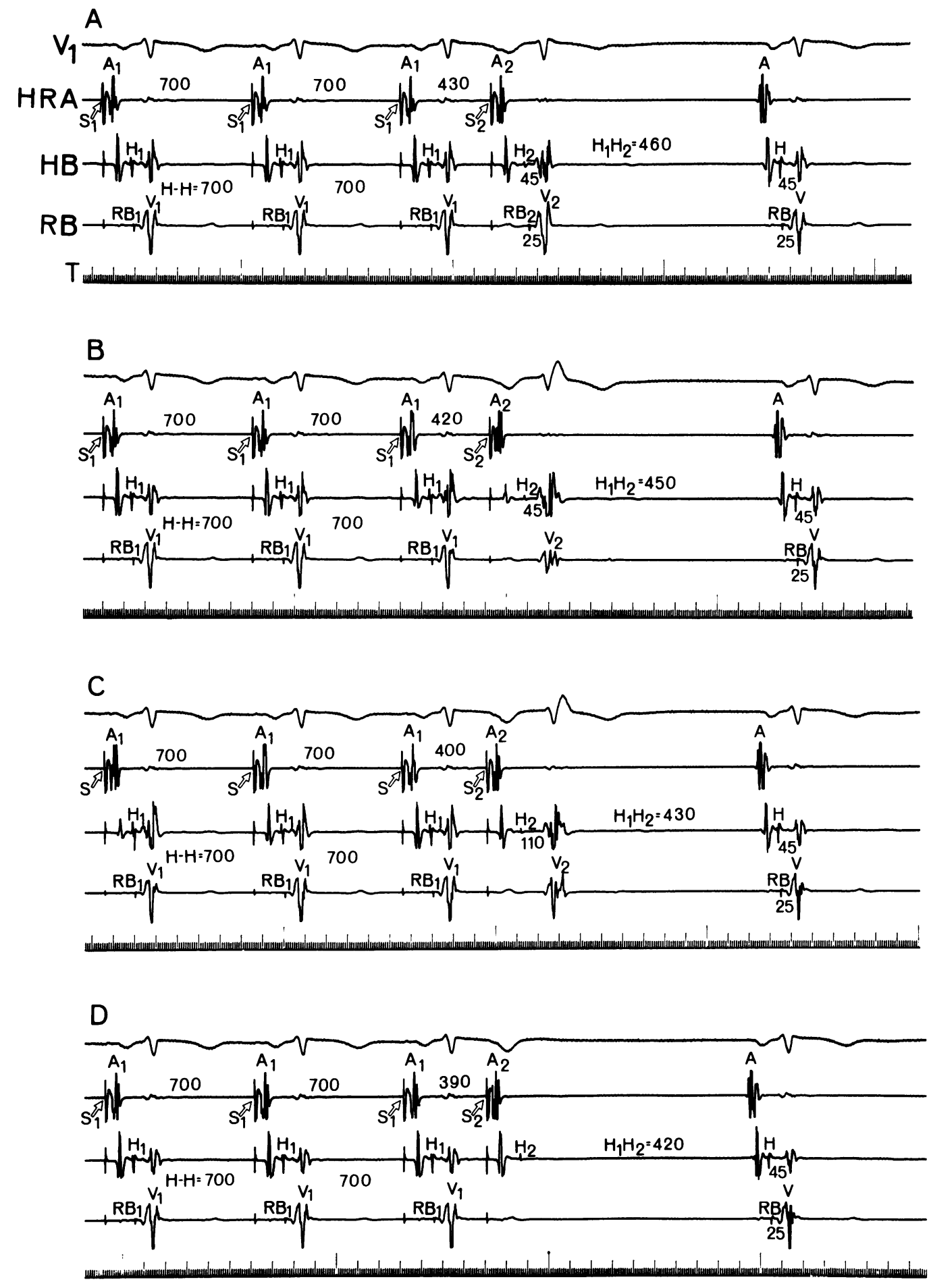

Figure 5. Illustration of method I from patient No. 8. For all panels $B C L$ is $700 \mathrm{~ms}$ and $\mathrm{HV}$ and RB are $\mathbf{4 5}$ and $25 \mathrm{~ms}$, respectively, on reference sinus beats. $A$ shows no VAb at $\mathrm{H} 1 \mathrm{H} 2$ of $460 \mathrm{~ms}$. With a decrease by $10 \mathrm{~ms}$ in $\mathrm{H} 1 \mathrm{H} 2$ interval functional RBBB occurs $(B)$. Note that $\mathrm{H} 2 \mathrm{~V} 2$ has remained at $45 \mathrm{~ms}$, but RB2 potential is no longer present. Further decrease in $\mathrm{H} 1 \mathrm{H} 2$ to 430 ms results in functional RBBB with increase in $\mathrm{H} 2 \mathrm{~V} 2$ to $110 \mathrm{~ms}$. Again no $R B 2$ potential is evident (C). In $D$ bilateral BB below $\mathrm{HB}$ occurs at an $\mathrm{H} 1 \mathrm{H} 2$ of $420 \mathrm{~ms}$.

of VAb noted during this study, i.e., RBBB alternating with no VAb, RBBB alternating with RBBB, RBBB alternating with LBBB, and BBH alternating with $\mathrm{BBH}$. It will also need to account for the patterns of $\mathrm{HV}$ conduction delays observed with $\mathrm{VAb}$ and why certain patterns of VAb, i.e., LBBB alternating with LBBB were not evident. 

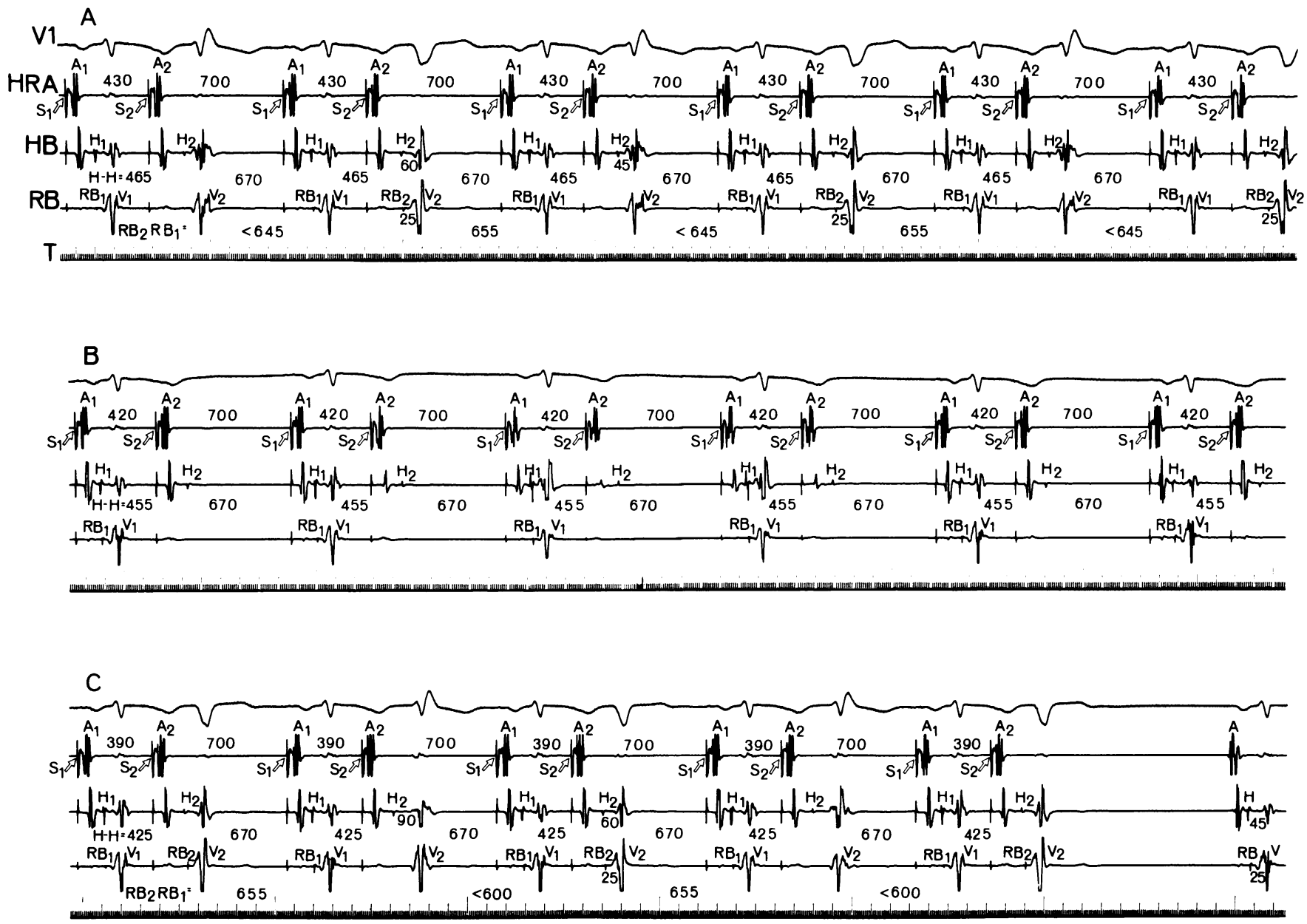

Figure 6. These tracings are from the same patient as in Fig. 5 but depict method II. Note the shorter "long" H-H CL with this method compared with method I in Fig. 5 despite the identical long atrial CL. In $A$ at an H1H2 of 465 ms both functional RBBB and LBBB occur in an alternating pattern. This is a longer coupling interval than onset of functional RBBB during method I. Moreover, functional LBBB was never manifest during method I. Note that during functional RBBB the $\mathrm{HV}$ interval remains at $45 \mathrm{~ms}$ but no RB potential is recorded, whereas during functional LBBB the HV interval is $60 \mathrm{~ms}$ and the RB-V interval is equal to the reference sinus beat
(C) at $25 \mathrm{~ms}$. In $B$ bilateral BBB below $\mathrm{HB}$ occurs at an $\mathrm{H} 1 \mathrm{H} 2$ of 455 ms compared with 420 ms during method I (Fig. $5 \mathrm{D}$ ). With further $\mathrm{H} 1 \mathrm{H} 2$ abbreviation HPS conduction again occurs with alternating functional RBBB and LBBB at identical H1H2. However, there is now HV prolongation with both functional RBBB and LBBB (compared with $A$ ) and prolongation is greater with functional RBBB. Again note the absent of RB potential during functional RBBB and the same RB-V interval during functional LBBB compared with the reference beat.
Results from this study as well as previous reports indicate that the site of conduction delay and/or block during VAb with $A 2$ is usually between $\mathrm{H}$ and $\mathrm{BB}$ recording sites, probably in the proximal $\mathrm{BBs}(3,17,23,24)$, although the present study cannot exclude a site within the HB (with longitudinal dissociation (25-28). Studies in man have suggested (17, 29-32), and canine studies by Moe et al. (3) have shown that where A2 results in functional $\mathrm{RBBB}, \mathrm{RB}$ could be depolarized retrogradely via transeptal activation from impulse propagated by LB. There is therefore a significant delay in time of depolarization distal to the site of block ( $30 \mathrm{~ms}$ in canine hearts). This then sets the stage for a similar decrease in CL at this site within the HPS with the next Al where every other beat is "long" as in the present study. Such a CL change could shorten refractoriness at this site, abolishing VAb with the next A2. The reverse sequence of $\mathrm{CL}$ changes at the initial site of block would then occur, i.e., a relative increase in CL at this site within the HPS with the next $A 1$ resulting in an increase in refractoriness and manifestation of VAb with the next A2. Such an effect would result in a persistent pattern of RBBB alternating with no VAb 
Table II. Patterns of VAb on Alternate A2 during Method II (Longest H1H2)

\begin{tabular}{ccccccc}
\hline Patient & NL/NL & R/NL & R/R & R/L & BBH/BBH & $\begin{array}{l}\text { Onset of HV } \\
\text { prolongation }\end{array}$ \\
\hline No. & & & & & & \\
1 & 530 & - & - & 520 & 470 & 520 \\
2 & 520 & 510 & - & - & - & - \\
3 & 445 & - & - & 430 & - & 430 \\
4 & 395 & - & - & 385 & - & 385 \\
5 & 405 & 400 & - & - & - & - \\
6 & 415 & 405 & 355 & - & - & - \\
7 & 450 & 440 & - & - & - & - \\
8 & 470 & - & - & 465 & 455 & 465 \\
9 & 500 & 480 & 470 & 450 & 420 & 450 \\
10 & 460 & 450 & - & - & - & - \\
11 & 450 & - & - & 430 & - & 430 \\
12 & 580 & 570 & - & 520 & - & 520 \\
13 & 510 & 495 & - & - & 465 & - \\
14 & 500 & 495 & - & - & - & - \\
\hline
\end{tabular}

All electrophysiologic data are given in milliseconds. Values (other than for NL/NL) are given for onset of a given pattern of VAb. NL/ $\mathrm{NL}$; normal intraventricular conduction (NL) alternating with $\mathrm{NL}$; R/NL, RBBB alternating with NL; R/R, RBBB alternating with RBBB; R/L, RBBB alternating with LBBB; BBH/BBH, Block below His bundle $(\mathrm{BBH})$ alternating with $\mathrm{BBH}$. as was seen in this study. Where $\mathrm{A} 1 \mathrm{~A} 2$ (and $\mathrm{H} 1 \mathrm{H} 2$ ) is further abbreviated, the shortening in CL described above following $\mathrm{VAb}$ with $\mathrm{RBBB}$ may not be sufficient to abolish refractoriness and RBBB may occur with each A2.

While more complex, alternating RBBB and LBBB VAb may be similarly explained. In such instances refractoriness in both $\mathrm{BBs}$ (or $\mathrm{H}-\mathrm{BB}$ axes) is encountered with $\mathrm{A} 2$ in the form of conduction delay or block in the BB. In the event of block the BB manifesting the block is depolarized retrogradely from the contralateral $\mathrm{BB}$ and therefore significantly shortens the next BB2BB1 CL compared with the contralateral BB. Ipsilateral BB CL shortening will also take place if A2 simply shows delay rather than block within the BB distal to the site where delay starts. The resulting decrease in refractoriness of the ipsilateral BB favors the occurrence of contralateral BBB with the next A2. In a sense, alternating $A 2$ unmasks refractoriness in the contralateral $\mathrm{BB}$ and probably explains the higher incidence of both $R$ and LBBB occurring in the same patient where VAb manifests with $\mathrm{HV}$ prolongation with this method compared with constant CL. Such changes in BB CL should produce alternating patterns of functional BBB ad infinitum as was observed in this study.

It was also observed that during alternating BBB the HV prolongation was either present during both RBBB and LBBB or only during $\mathrm{LBBB}$. A possible explanation for this may relate to the differential effect on refractoriness of BB CL shortening following VAb at and/or distal to the site of block on H-RB

Table III. Effect of Alternating Changes in Atrial CL with VAb on Bundle Branch CL

\begin{tabular}{|c|c|c|c|c|c|c|c|c|c|c|c|c|c|c|}
\hline \multirow[b]{2}{*}{ Patient } & \multirow[b]{2}{*}{ H-V } & \multirow[b]{2}{*}{ RB-V } & \multicolumn{6}{|c|}{ A2 (odd beats) } & \multicolumn{6}{|c|}{ A2 (even beats) } \\
\hline & & & $\begin{array}{l}\text { Type block } \\
\text { HPS }\end{array}$ & $\mathrm{H} 2 \mathrm{H} \mathrm{I}$ & $\mathrm{H} 1 \mathrm{H} 2$ & $\mathrm{H} 2 \mathrm{~V} 2$ & RB2V2 & RB2RB1 & $\begin{array}{l}\text { Type block } \\
\text { HPS }\end{array}$ & $\mathrm{H} 2 \mathrm{H} \mathbf{l}$ & H1H2 & $\mathrm{H} 2 \mathrm{~V} 2$ & RB2V2 & RB2RB1 \\
\hline \multicolumn{15}{|l|}{ No. } \\
\hline 1 & 50 & 15 & RBBB & 680 & 520 & 50 & $<0$ & 660 & LBBB & 680 & 520 & 70 & 15 & $<665$ \\
\hline 2 & 45 & 10 & RBBB & 750 & 510 & 45 & $<0$ & 750 & None & 750 & 510 & 45 & 10 & $<740$ \\
\hline 3 & 55 & 30 & RBBB & 640 & 430 & 55 & $<0$ & 625 & LBBB & 640 & 430 & 70 & 30 & $<610$ \\
\hline 4 & 55 & 25 & RBBB & 620 & 385 & 70 & $<0$ & 595 & LBBB & 620 & 385 & 80 & 25 & $<580$ \\
\hline 5 & 50 & 25 & RBBB & 610 & 400 & 50 & $<0$ & 610 & None & 610 & 400 & 50 & 25 & $<585$ \\
\hline 6 & 45 & 20 & RBBB & 580 & 405 & 45 & $<0$ & 580 & None & 580 & 405 & 45 & 20 & $<560$ \\
\hline 7 & 45 & 25 & RBBB & 760 & 440 & 45 & $<0$ & 760 & None & 760 & 440 & 45 & 25 & $<735$ \\
\hline \multirow[t]{2}{*}{8} & 45 & 25 & RBBB & 670 & 465 & 45 & $<0$ & 655 & LBBB & 670 & 465 & 60 & 25 & $<645$ \\
\hline & & & RBBB & 670 & 425 & 90 & $<0$ & 655 & LBBB & 670 & 425 & 60 & 25 & $<600$ \\
\hline \multirow[t]{2}{*}{9} & 45 & 10 & RBBB & 680 & 480 & 45 & $<0$ & 680 & None & 680 & 480 & 45 & 10 & $<670$ \\
\hline & & & RBBB & 670 & 450 & 45 & $<0$ & 610 & LBBB & 670 & 450 & 105 & 10 & $<660$ \\
\hline
\end{tabular}

All electrophysiologic data are given in milliseconds. 
vs. H-LB axis. Since HV interval is a measurement of the conduction time between onset of $\mathrm{HB}$ and ventricular depolarization the results of this study would suggest that a greater magnitude of conduction delay occurs along the H-RB axis with LBBB than occurs along the H-LB axis with RBBB. This is suggested by the longer $\mathrm{HV}$ interval associated with LBBB compared with RBBB except in one patient (No. 8) where further $\mathrm{H} 1 \mathrm{H} 2$ shortening produced a gap phenomenon within the HPS (10). While a higher incidence of $\mathrm{HV}$ prolongation with LBBB compared with RBBB VAb has been previously observed during $A 2$ technique with constant BCL (13), conditions in the present study are modified such that during VAb the subsequent ipsilateral BB CL is shortened. It would appear that at the $\mathrm{H} 1 \mathrm{H} 2$ range where alternating functional RBBB and LBBB manifests, CL shortening along the H-RB axis following RBBB is not sufficient to abolish refractoriness along this axis (resulting in $\mathrm{HV}$ prolongation with subsequent LBBB). However, shortening of H-LB axis refractoriness following LBBB may or may not be sufficient (as indicated by the absence or presence of $\mathrm{HV}$ prolongation with subsequent $\mathrm{RBBB}$ ). These findings are compatible with shorter refractoriness of the H-LB axis for the CLs tested in these patients and probably explains why LBBB VAb alone with every A2 was never observed (as compared with $\mathrm{RBBB}$ $\mathrm{VAb}$ ). Instead further $\mathrm{H} 1 \mathrm{H} 2$ shortening results in bilateral block within the HPS.

In conclusion, the observations of the present study suggest that during circumstances of alternating $C L$ changes additional factors besides the $\mathrm{H} 1 \mathrm{H} 1$ and $\mathrm{H} 1 \mathrm{H} 2$ relationships must be evaluated to predict onset of conduction delay and/or block within the HPS as well as the particular patterns of VAb. These factors include the dynamic response of the HPS to abrupt alterations in $\mathrm{CL}$ and the effect of VAb on subsequent ipsilateral bundle branch cycle length.

\section{Acknowledgments}

We wish to thank Ms. Ann Edwards and Mr. Brian Miller for their assistance in the preparation of this manuscript.

\section{References}

1. Lewis, T. 1912. Observations upon disorders of the heart's action. Heart. 3:279-300.

2. Katz, L. M., and A. Pick. 1956. Clinical Electrocardiology: Part I. The Arrhythmias. Lea \& Febiger, Philadelphia. 250.

3. Moe, G. K., C. Mendez, and J. Han. 1965. Aberrant AV impulse propagation in the dog heart. A study of functional bundle branch block. Circ. Res. 16:261-286.

4. Cohen, S. I., S. H. Lau, J. I. Haft, and A. N. Damato. 1967. Experimental production of aberrant ventricular conduction in man. Circulation. 36:673-685.

5. Cohen, S. I., S. H. Lau, B. J. Scherlag, and A. N. Damato. 1969. Alternate patterns of premature ventricular excitation during induced atrial bigeminy. Circulation. 39:819-829.
6. Wit, A. L., M. B. Weiss, W. D. Berkowitz, K. M. Rosen, C. Steiner, and A. N. Damato. 1970. Patterns of atrioventricular conduction in the human heart. Circ. Res. 27:345-359.

7. Damato, A. N., P. J. Varghese, A. R. Caracta, M. Akhtar, and S. H. Lau. 1973. Functional 2:1 AV block within the His-Purkinje system: simulation of type II second-degree AV block. Circulation. 47:534-542.

8. Akhtar, M., A. N. Damato, A. R. Caracta, W. P. Batsford, M. E. Josephson, and S. H. Lau. 1974. Electrophysiologic effects of atropine on atrioventricular conduction studied by His bundle electrogram. Am. J. Cardiol. 33:333-343.

9. Denes, P., D. Wu, R. Dhingra, R. J. Pietras, and K. M. Rosen. 1974. The effects of cycle length on cardiac refractory periods in man. Circulation. 49:32-41.

10. Akhtar, M., A. N. Damato, W. P. Batsford, A. R. Caracta, G. Vargas, and S. H. Lau. 1974. Unmasking and conversion of gap phenomenon in the human heart. Circulation. 49:624-630.

11. D'Cruz, I. A., H. Cohen, A. Pick, and R. Langendorf. 1975. Mechanisms of electrical alternans in left and right bundle block. Early or late phases of refractoriness in the bundle branch system. Am. J. Cardiol. 35:131. (Abstr.)

12. Reddy, C. P., A. N. Damato, M. Akhtar, M. B. Dhatt, J. A. Gomes, and J. R. Foster. 1978. Study of the temporal effects on conduction and refractoriness of the His-Purkinje system in man. Am. Heart J. 96:316-325.

13. Denker, S. T., C. J. Gilbert, M. Shenasa, and M. Akhtar. 1983. An electrocardiographic-electrophysiologic correlation of aberrant ventricular conduction in man. J. Electrocardiol. (San Diego). 16:269-277.

14. Denker, S., M. Shenasa, C. J. Gilbert, and M. Akhtar. 1983. Effects of abrupt changes in cycle length on refractoriness of the HisPurkinje system in man. Circulation. 67:60-68.

15. Scherlag, B. J., S. H. Lau, R. H. Helfant, W. D. Berkowitz, E. Stein, and A. N. Damato. 1969. Catheter technique for recording His bundle activity in man. Circulation. 39:13-18.

16. Akhtar, M., C. Gilbert, F. Wolf, and D. H. Schmidt. 1978. Reentry within the His-Purkinje system. Elucidation of reentrant circuit utilizing right bundle branch and His bundle recordings. Circulation. 58:295-304.

17. Akhtar, M., C. Gilbert, M. Al-Nouri, and S. Denker. 1980. Site of conduction delay during functional block in the His-Purkinje system in man. Circulation. 61:1239-1248.

18. Lau, S. H., G. A. Bobb, and A. N. Damato. 1970. Catheter recording and validation of left bundle-branch potentials in intact dogs. Circulation. 42:375-383.

19. Rosen, K. M., S. H. Rahimtoola, M. Z. Sinno, and R. M. Gunnar. 1971. Bundle branch and ventricular activation in man. A study utilizing catheter recordings of left and right bundle branch potentials. Circulation. 43:193-203.

20. Langendorf, R. 1951. Aberrant ventricular conduction. Am. Heart J. 41:700-707.

21. Denker, S., M. H. Lehmann, R. Mahmud, C. Gilbert, and M. Akhtar. 1983. Divergence between His-Purkinje system and ventricular muscle refractoriness with abrupt changes in cycle length. Circulation. 68:1212-1221.

22. Denker, S., M. Lehmann, R. Mahmud, C. Gilbert, and M. Akhtar. 1984. Facilitation of macro reentry within the His-Purkinje system with abrupt changes in cycle length. Circulation. 69:26-32.

23. Zipes, D. P., R. F. Knope, C. Mendez, and G. K. Moe. 1975. 
The site of functional right bundle branch block in the intact canine heart. Adv. Cardiol. 14:105-114.

24. Glassman, R. D., and D. P. Zipes. 1981. Site of antegrade and retrograde functional right bundle branch block in the intact canine heart. Circulation. 64:1277-1286.

25. Anderson, G. J., K. Greenspan, J. P. Bandura, and C. Fisch. 1970. Asynchrony of conduction within the canine specialized Purkinje fiber system. Circ. Res. 27:691-703.

26. Myerburg, R. J., K. Nilsson, B. Befeler, A. Castelanos, and H. Gelband. 1973. Transverse spread and longitudinal dissociation in the distal A-V conducting system. J. Clin. Invest. 52:885-895.

27. Narula, O. S. 1977. Longitudinal dissociation in the His bundle. Bundle branch block due to asynchronous conduction within the His bundle in man. Circulation. 56:996-1006.
28. El-Sherif, N., F. Amat-y-Leon, C. Schonfield, B. J. Scherlag, K. Rosen, R. Lazzara, and C. Wyndham. 1978. Normalization of bundle branch block patterns by distal His bundle pacing. Clinical and experimental evidence of longitudinal dissociation in the pathologic His bundle. Circulation. 57:473-483.

29. Gouaux, J. L., and R. Ashman. 1947. Auricular fibrillation with aberration simulating ventricular paroxysmal tachycardia. Am. Heart J. 34:366-373.

30. Langendorf, R., and A. Pick. 1975. Concealed intraventricular conduction in the human heart. Adv. Cardiol. 14:40-50.

31. Mazzoleni, A., D. Johnson, E. Fletcher, and R. N. Class. 1972. Concealed conduction in left bundle of His. Br. Heart J. 34:365-369.

32. Neuss, H., M. Schlepper, and J. Thormann. 1975. Analysis of reentry mechanisms in three patients with concealed Wolff-ParkinsonWhite syndrome. Circulation. 51:75-81. 